grandi quantilà, e gli Autori non hanno potuto avere a loro disposizione che pochi grammi (10 a $12 \mathrm{gr}$.) di carburo ottenuto per sintesi.

Rimane adunque incerto se il propiltaluene sia identico o no col cimene; ma, secondo il parere di Fittig, e dei suoi colleghi questa incertezza sparirà quando si conoscerà positivamente la costituzione del cumene. Difalli se quest'ultimo é, come essi ritengono per cosa probabilissima, isopropilbenzina; il cimene, secondo la maniera della sua formazione, deve esserc considerato come isopropilmetilbenzina ; diverso ciod dal propiltoluene ora preparato, o propilmetil benzina.

\title{
SUNTI DI LAVORI DI FISICA
}

Intorno al limite di attivita delle formo molecolari di capillaritá; per G. QUINCKE. ( Nachrichten d. d. $k$. Gesellschaft d. Wissenschaften u. d. G. A. Universität zu Göllingen, Maggio 1869). - I fenomeni di capillariti si spicgano, come è noto, ammettendo forze molecolari che agiscano soltanto a disianze insensibili. Ma dalle esperienze di Simon, Bède, Werthein e Wilhelmy risultano valori diversi delle costanti di capillarità per lo stesso liquido a seconda della curvatura delle pareti solide; e sembra cho ciò non si possa spiegare se non si ammette clie non sia assolutamente insensibile la distanza massiona alla quale agiscono le forze molecolari, ossia il cosiddetto raggio della sfera di attiviti, che nel seguito s'indicherà sempre con $l$.

Plalcau, per quanto è a cognizione dell' A., cercò pel primo di determinare la quantità $l$ amınettendo che una sottile lamina di glicerina non possa piu sussistere appena che abbia raggiunto una grossezza inferiore a $2 l$. Cosi trovò $l=0 \mathrm{~mm}, 0000567$. Leidenforsl trovò per la grossezzil minima di

Sario. 2. Vol. II. 
una lamina di acqua saponata $u$ mm,001772. Mach trovò pel vetro liquido $0^{\text {num }}, 142$, per la colofonia fusa 0 :nm, 027 .

Ma I'A. prescindendo anche dalle obbiezioni teoriche, pensa che questo znetodo debba fornire per $l$ dei valori mag. giori del vero per la difficolta di preparare appunto la lamina di minima grossezza, e perciò ha seguito un altro metodo che permette di determinare $l$ direttamente.

Ricopre la superficie ben tersa ed omogenea di una lastra di vetro con uno strato cuneiforme d' un' altra sostanza, la cui grossezza sia pressochè nulla sul taglio del cuneo e vada via via aumentando.

Quando si metta a contatto questo strato cuneiforme con un liquido che non lo bagni, l'angolo $w$ col quale la superfi cie liquida incontra la parete solida dipenderà dall' attrazione delle particelle della parcte su quelłe del liquido e sarà costante solo iu corrispondenza ad una grossezza dello strato maggiore di $l$. Per grossezze minori deve influirc sul liquido anche il vetro sottoposto e modificare il detto angolo.

L'A. versò del liquido di Morin per inargentare sopra un vetro piano e vi pose sopra un cilindro di vetro da specchi del raggio di 120min lisciandovi cosi depositare uno strato di argento a doppio cuneo che nel mezzo era più soltile (1).

Preparate cosi duc lastre di vetro, le sciacquò con acqua distillata e ve le tenne immerse per qualche tempo per allontanarne ogni traccia del liquido adoperato; e poi le pose di fronte dalle parti inargentate in modo che si corrispondessero grossezze uguali degli strati di argento e che fra loro rimanesse un piccolo intervallo. Cosi disposte, le immerse nell' acqua distillata tenendo verticali le linee di grossezza minime degli strati d'argento. Nello spazio capillare interposto alle due lamine, l'acqua si elevò ad un' allezza y la quale era maggiore là dove l' argento era più soltile, ed andava scemando dai due lati. L' A. misuro l'altezza media $y$ a distanze $x$ diverse dal punto di massima sottigliezza. Poi cambiò l'argento in ioduro argentico e dál colore di questo col

(1) V. Pogg. Ann. 129, p. 184. 
metodo di Fizeau calcolo le grossezze dell'argento nei diversi punti (1). Nei punti più grossi, ancora trasparenti, potè sempre notare un decrescimento di $y$ ossia un aumento dell'an. golo $w$ poichè :

$$
\cos w=\frac{y \mathrm{E}}{a^{2}}
$$

essendo $\mathrm{E}$ la distanza delle lastre, $a^{2}$ la costante di Poisson o la cosiddetta cocsione specifica del liquido. $\Lambda$ seconda della costituzione molecolare dell'argento, questa grossexza oscillava fra $0^{\mathrm{mn}}, 000036$ e $0^{\mathrm{mmm}}, 0000542$, cosicchè $\mathrm{l}^{\prime} \mathrm{A}$. riliene per il vetro, l'argento e l'acqua $l>0$ inro,0000:

Osserva però che l' esperienza non è di sicura riuscita, specialmente impiegando soluzioni d'argento preparate di fresco, poichè spesso l' acqua sta alla medesima altezza qualunque sia la grossezza dello strato di argento.

L'A. lascio poi che lo lastre inargentate si asciugassero dopo averle ben lavate coll'acqua distillata ed allora trovo differenti elevazioni in corrispondenza alle diverse grossez.ze dello strato d'argento; e la curva formata dal menisco variare col tempo durante il quale le lastre rimasero asciutte, cosicchè l'A. opina che ne sia cagione la maggior'e o uninor quantità d'aria assorbita dall argento alla sua superficie. E siccome coi mezzi ottici non potè notare verun foro nello strato d'argento, cosi conchiude che la quantità d' aria assorbita deve dipendere dalla grossezza dell' argento ed influire sull'elevazione capillare. Perciò il valore di $l$ trovato superiormente determinerebbe iu questo caso il raggio d'attivita delle forze molecolari d'assorbimento.

Pei liquidi opachi e specialmente pel mercurio si può misurare facilmente l'angolo $w$ formato con una parete solida trasparente per mezzo della luce riflessa dall'ullimo elemento della superficie liquida. Si abbassa l'occhio fino a che l'immagine di una famma p. e., scompaia; e quanto più si deve abbassare tanto minore è l' angolo formato dalla superficie del mercurio colla parete, ossia il supplemento di $w$.

(1) V. Pogg. Ann. 129, p. 181 . 


\section{4}

L'A. tagliò col diamante una lastra inargentata, quale fu descritta, perpendicolarmente alla linea di minima grossezza dello strato d'argento. Uno dei pezzi fu trallato coll'idrogeno solforato e così l'argento trasformato in solfuro argentico, l'argento dell' altro pezzo fu cambiato invece in ioduro argentico. Il primo pezzo fu immerso verticalmente nel mercurio e col metodo ora cilato si osservò come l'angolo $w$ decresceva poco a poco a partire dai punti più sottili dal solfuro argentico fino a che assumeva un valore costante.

L'A. determinò poi il colore proprio al punto corrispondente dell' altro pezzo di lastra coperta di ioduro. Indicando con $\varepsilon$ la grossezza dello strato d'aria proprio a quell' anello di Newton avente lo stesso colore, con D la grossezza, con $\sigma$ il peso specifico, con $\alpha$ l'equivalente, e con $n$ l'indice di refrazione del solfuro argentico ; ed indicando colle stesse lettere affette da un apice le stesse quantita relative all' iodaro di argento, si ha :

$$
\mathrm{D}=\frac{\alpha}{\alpha_{1}} \frac{\sigma_{1}}{\sigma} \frac{\varepsilon}{n_{1}}=0,1918 \varepsilon
$$

avendo posto :

$$
\begin{array}{ll}
\alpha=123,9 & \sigma=6,350 \\
\alpha_{1}=234,9 & \sigma_{1}=5,602
\end{array} \quad n_{1}=2,25
$$

Il colore dell' ioduro nel punto corrispondente al punto del solfuro, in cui l'angolo $w$ cominciava ad essere costante, era in media giallo oscuro, ossia aranciato-rossastro di $1 .^{\circ}$ ordine veduto per riflessione; quindi $\varepsilon=0 \mathrm{~mm}, 000252$ e per conseguenzo $\mathrm{D}=0^{\mathrm{min}}, 00004833$. - $\mathrm{L}^{\prime} \mathrm{A}$. prende dunque pel vetro, pel solfuro d' argento e pel mercurio :

$$
l=0^{\mathrm{mm}, 00004833} \text {. }
$$

Del resto le variazioni dell'angolo $v$ si possono anche dedurre dall'altezza di $z_{1}$ alla quale discende il mercurio presso la parte solida, poichè la teoria dà $z_{1} \Rightarrow a \cos w$. 
I. A. osserva che si possono istituire esperienze simili con altre sostanze depositate sul vetro; ma esse, come l'ioduro $d^{\prime}$ argento, vengono attaccate dal mercurio, e d'altra parte torna molto difficile determinarne la grossezza con soddisfacente precisione. Tuttavia siccome si può rilevare l'angolo $w$ con molta celcriti, così l' A. non mancò di osservarlo anche per l'ioduro argentico e trovó che cominciava ad esser costante di fronte ad una grossezza dell' ioduro di 0um, 0000 sy cosicchè per il vetro, l' ioduro d'argento ed il mercurio dà :

$$
l=0^{\mathrm{mm}}, 000059 \text {. }
$$

Per le sostanze poco refrangenti, come il collodio, $v^{\prime} \grave{e}$ l' inconveniente che le grossezze, per le quali l'angolo col mercurio si fa costante, appaiono nere nella luce riflessa. Siccome l'indice di refrazione del collodio $(1,369)$ è più piccolo di quello del vetro, la grossezza d'aria corrispondente darebbe il bianco di $10^{\circ}$ ordine, e la grossezza dello strato di collodio sarebbe di $\frac{0^{\mathrm{mit}}, 000109}{1,369}$ e per couseguenza

$$
l<0,0000797 .
$$

L'A. ha tentato di produrre uno strato cunciforme di selenio riducendo dell'acido selenioso con una fiamma alla Bunsen e tenendovi sopra una lastra di vetro fredda. Anche in questo caso l'angolo $w$ risultava variabile quando la lastra era immersa nel mercurio e pareva che conducesse a valori di $l$ poco discosti dai precedenti, quantınque all' $\Lambda$. non riuscisse di determinare esattamente la grossezza dello strato di selenio.

L'A. non nasconde la possibilità che l'angolo $w$ sia reso variabile non dallo strato cunciforme, ma dall' aria assorbita da esso, quantunque ciò gli sembri molto improbabile di fronte ai risultati concordi delle varic esperienze. $E$ quand'anche fosse, il valore trovato di $l$ determinerebbe sempre il raggio d'atlivita delle forze molecolari di assorbimento.

Stando agli esperimenti ora accennati la distanza massi- 
ma, alla quale le forze molecolari sono ancora attivc, sarebbe di 50 millionesimi di millimetro, o all' incirca un decimo della lungheza d' onda della luce avente una refrangibilita media. Tale distanza è maggiore di quanto se l' aspeltava l'A.; ed egli opina che anche altre forze molecolari, come quelle determinanti l' elasticità e le proprietà oltiche dei corpi, agiscano similmente a distanze fiuile, ciò che dovrebbe modifi. care notevolmente le vedute teoriche che ora si ammettono.

\section{A. Rorti.}

Eicorche Eull' illumingzione doi liquidi por mezzo d' un fascio di Ince noutra o polariz. zata; per M. LALLEMAND. (Comples Rendus, 19 Luglio 1869 ). - Dal punto di vista degli effetti ottici prodotti da un fascio di luce solare nei differenti liquidi, l'Autore c condotto a dividere $i$ liquidi in tre categorie: quelli che non hanno fluorescenza apprezzabile, nè potere rotatorio - quelli dotati di fluorescenza, ma che non esercitano veruna deviazione sul piano di palisizzazione della luce incidente - quelli che hanno potere rotatorio ed anche una fuorescenza alquanto energica. Ciò premesso, espone i risultati delle sue esperienze sopra ciascuna di queste categoric di liquidi, e ne deduce delle conchiusioni importanti.

Il liquido è introdolto in un grosso tubo di vetro chiuso alle estremità da due lastre parallele. Il tubo è disposto orizzontalmente in una camera oscura e riceve nella direzione dell' asse un fascio di raggi solarj riflessi da uno specchio metallico e resi leggiermente convergenti da una lente acroInatica a lungo fuoco; un prisma di Foucault può essere posto sul tragitto del fascio per polarizzarne la luce in un piano determinato.

Sia il tubo pieno d'acqua pura e sia altraversato dalla luce neutra. Guardandolo in una direzione trasversale $l^{\prime}$ A. ha constatato che l'acqua si illumina $e$ dirigendo un prisma di Nicol normalmente all' asse del fascio, ha verificato che la estinzione e completa quando la sezione principale del prisma è parallela all' asse del tubo; cioc̀ la luce, emessa dall'acqua in una direzione qualunque normale all' asse del fir- 
scio, $\grave{e}$ interamente polarizzata in un piano che passa per quell' asse. Inclinando il Nicol più o mevo su quella dirczio. ne, l estinzione è più o meno completa.

Sia ora la luce incidente polarizzata in un piano oriz2ontale: In tal caso il tubo non appare illuminato che in direzione orizzontale, ed appare assolutamente oscuro guardato che sia dall' alto al basso. Si riproduce così l'esperienza suggerita da Stokes a Tyndall nelle sue ricerche sulle condensazioni nebulose che la luce clettrica produce in un mezzo rarefatlo contenente dei vapori decomponibili. Ma, secondo l'A., la conchiusione che se ne deduce è ben diversa. Mentre nell' esperienza di Tyudall, l' illuminazione si attribuisce ad un fenomeno di riflessione sopra particelle solide o liquide estremamente tenui; con un mezzo trasparento ed omogeneo quale è l' acqua distillata di recente, non si può più invocare un effetto di riflessione speciale; ma si deve ritenere una vera propagazione del movimento vibratorio nell'etere condensato del mezzo refrangente che si effettua soltanto in tutte le direzioni del piano di polarizzazione della luce incidente: e ciò si prova con un Nicol analizzatore. Po. nendo il Nicol orizzontalmente ed in direzione normale all' asse del tubo, si verifica che la luce emessa trasversalmente e completamente polarizzata, ed inclinando l'analizzatorc sull'asse del tubo si riconosce che sollo ogni incidenza la luce emessa $\grave{c}$ sempre interamente polarizzala in un piano orizzontale.

Le due esperienze ora riferite sono anzi tullo una verificazioue del principio di Huyghens cui si ricorre in particolar modo per ispiegare i fenomeni della diffrazione; e l'A. crede che la seconda di esse sia una prova visibile della direzione del movimento vibratorio dell etere in un raggio polarizzato. Dai fenomeni fin qui osservati si era condotti a ritenere che le molecole d'etere vibrassero nel piano dell'onda $e$ che in un raggio polarizzato le vibrazioni fossero rettilinee e perpendicolari al raggio, ma non si poteva ancora decidere se queste vibrazioni fossero parallele o normali al piano di polarizzazione. Ma nell' esperienza citata si vede che il moto vibratorio non si propaga normalmente al piano e 
128

si propaga invece in tutte le direzioni del piano di polarizzazione. Dunque, dice l'A., le molecole d'etere vibrano normalmente a questo piano ed in tale direzione non possono propagare che delle onde analoghe alle onde aeree le quali, se anche esistono, non producono alcun fenomeno luminoso.

L'acido nitrico, l'acido cloroidrico, l' ammoniaca, cc. si comportano come l'acqua; ed il fenomeno riesce molto più appariscente col collodio preparato di fresco, ben limpido ed incoloro.

Coi liquidi fluorescenti si oltengono risultati più complessi. D" ora in poi il fascio luminoso si supporra sempre polarizzato orizzontalmente. Con una soluzione diluitissima di esculina o di solfato di chinina, guardando il tubo verticalmente si vede una tinta turchina uniforme la cui intensità va scemando dalla faccia d' incidenza all'altro estremo. Questa luce è neutra all' analizzatore. Guardando orizzontalınente, $l$ 'illuminazione ed turchina all' origine $\mathrm{e}$ ben presto diventa bianca ed anzi all'estremo opposto è alquanto giallastra. Il Nicol mostra che questa luce è parzialmente polarizzata nel piano primitivo, e nella posizione d' estinzione persiste una tinta turchina identica a quella che si osserva direttamente nel medesimo punto guardando dall'allo al basso. Questo processo offre un mezzo comodo d'isolare e d'analizzare la illuminazione esclusivamente dovuta alla fluorescenza.

Se invece si dispone davanti al tubo un recipiente con una coluzione più carica di esculina o di solfato di chinina, sono traltenuti i raggi eccitatori violetti ed ultra-violetti, ed il liquido del tubo si comporta precisamente come l'acqua pura.

Questo processo d'analisi conduce a conseguenze inaspettate e mostra che la fluorescenza è molto più comune ai liquidi, di quello che si supponeva. Se non fu notata, dice l'A., in un gran numero di liquidi che la posseggono, ciò proviene dall' essere, in certi casi, tulti i raggi dello spetlro atti a provocare il fenomeno, e dal manifestarsi la fluorescenza del liquido in tutta la sua massa senza colore proprio ben deciso, invece che prodursi con maggiore evidenza e con un colore proprio alla superficie d'incidenza. Prende 
l'A. come esempio il solfuro di carbonio rellificato sulla calce e messo in contatlo di rame ridotto dall' idrogeno; in queste condizioni il liquido a perfettamente incoloro e, sottomesso all' azione dei raggi polarizzati, s' illumina per tutta la lunghezza del tubo e per tutte le direzioni di una linta bianca leggiermente azzurrognola. Guardando in direzione orizzontale con un polariscopio di Savart, vi si riconosce un poco di luce polarizzata; mentre che nella direzione verticale la luce emessa è neutra, interamente dovuta alla fluorescenza e composta di tutli i colori prismatici.

Operando con una luce omogenea qualsiasi, si trova che gli atomi nel solfuro di carbonio possono vibrare sotto $l^{\prime}$ influenza di tutti i raggi luminosi dello spettro ed emettere in seguito in tutte le direzioni della luce neutra di refrangibilità uguale o pochissimo differente. Molti liquidi d'origine organica e particolarmente i carburi d'idrogeno presentano gli stessi fenomeni.

Da quanto si è detto dell illuminazione dei liquidi per propagazione laterale direlta delle vibrazioni luminose, si possono prevedere $i$ singolari effetli che presentano $i$ liquidi dotati dal potere rotatorio quando siano sottomessi all'azione di un fascio polarizzato. Si prenda un lungo tubo pieno di una soluzione concentrata di zucchero di canna, e s' illumini da prima con una luce rossa omogenea ; siccome la soluzione non ha fluorescenza sensibile, cosi il tubo guardato verticalmente presso alla faccia d' incidenza, sembra oscuro. In direzione orizzontale emette all' incontro una luce intensa; ma coll' allontanarsi dall' incidenza, si osserva che bisogna girare allorno al tubo da sinistra a destra e guardare in direzione sempre più inclinata per vedere la fascia luminosa; e se si traccia sul tubo la direzione media di questa fascia, si ottiene un' elice il cui passo è precisamente uguale alla lunghezza della colonna di liquido attivo che farebbe compiere un' intera rivoluzione al piano di polarizzazione della luce incidente: il passo diminuisce col crescere della refrangibilita della luce incidente seguendo Ia legge approssimativa di Biot. Se la luce incidente è bianca, tutte le elici luminose, sovrapposte all' origine del tubo, si separano ben presto e 
danno un'illuminazione laterale prismatica di effetlo molto singolare. Se si dirige il raggio visualc da sinistra a destra, atlorno ad una data sezione del tubo, si vedono le tinte misle succedersi nell' ordine di refrangibilità. Guardando invece lungo una generatrice del cilindro a partire dalla sua origine, si osscrva la stessa successione delle tiute prismatiche. Ad onta delle previsioni teoriche che indicavano questo risultato, sorprende, dice l'A., di vedere completamente incoloro il fascio emergente dal tubo, mentre che le pareti brillano dei più vivi colori che variano secondo la dir ezione in cui si guardano. Sopprimendo il polarizzatore, tulte queste tinte scompaiono. L'essenza di trementina si conduce in modo analogo, colla differcaza che la rotazione visibile del piano di polarizzazione avviene da destra a sinistra, e che l'illuminazione laterale è complicata da una sensibile fluorescenza.

\section{A. RoITr.}

Sul riscaldamonto dei corpi solidi, caueato dal suono; per E. W ARBURG. (Berichte der $k$. Akademie $z u$ Berlin. Februar 1869). $\rightarrow$ Nel Vol. 24 degli Annali di Poggendorff Weber accenna al diverso tempo impiegato per le diverse sostanze poste in vibrazione, affinchè uu suono si estingua; dice che la resistenza dell' aria, la quale deve diminuire l'ampiezza d'oscillazione tanto più presto quanto minore è la massa del corpo, non basta a spiegare il fenomeno; e ne conchiude che debba dipendere dalla natura interna del corpo vibrante.

Infatti il piombo, quantunque molto più denso dell'acciaio, cessa di suonare molto più presto di questo.

Dietro tali considerazioni, una parte della forza viva di oscillazione deve impiegarsi nell' interno del corpo sonoro e probabilmente, dice l'A., trasmutarsi ivi in calore.

Inducono a considerazioni simili certi corpi che uniti ad altri corpi sonori causano uno smorzamento del suono da essi prodotto. Se sul prolungamento di un tubo di vetro si adatta un tubo di piombo, anche molto sottile, si trova che il tuono longitudinale reso dal tubo di vetro è molto ammorzato; e ciò avviene anche quando si dia al tubo di piom- 
bo la lunghezza di mezza onda, nel qual caso si ha un minimo d'ammorzamento. Una verga d'acciúio, o di ottone, in circostanze simili non produce effetto sensibile. Anche quesli fenomeni fauno supporre che una parte della forza viva di oscillazione s' impieghi nell' interno del corpo e che quindi vi avvenga uu riscaldamento, il quale sarà maggiore pel piombo che non per l'acciaio.

L'A. si propose di indagare sotto questo aspetto la produzione di calore per mezzo del suono; e perciò si servì di una coppia termoclettrica congiunta ad un galvanometro, ponendone la saldatura sui punti da esplorare dopo che il suono era cessato; ma essendosi previamente accertato che, prima del suono, quel contatto non deviava l'ago magnelico.

Suoni longitudinali. - Riusci prima di tutto a constatare un riscaldamento in uua verga di cera (sustanza che smorza il suono molto rapidamente). Questa verga era adatlata sul prolungamento di un tubo di vetro avendo la lunghezza di mezza onda calcolata dietro la velocita di propagazione del suono nella cera che l' $\Lambda$. ha determinato. Posta la saldatura della coppia termoclectrica al luogo di un nodo, ottenne una deviazione di 300 parti di scala nel verso del riscaldamento; pei ventri non l'iscontro che una deviazione di so p. nello stesso verso.

Un tubo di vetro avente $9^{\mathrm{mr}} \mathrm{di}$ diamelro esterno ed esso pure una lunghezza di mezza onda, adattato allo stesso tubo di vetro in luogo della cera, accusò al nodo un riscaldamento di 300 a 400 parti di scala, ed al ventre di 40 p. Un tubo di piombo più sottile ( $/ \mathrm{mm}^{\mathrm{m}}$ diam. ester.) ma della stessa lunghezza, unito allo stesso tubo di vetro, si riscaldo molto più dasdo una deviazione di $600 \mathrm{p}$. quando, dopo il suono, la saldatura cra sul nodo. Congiunti poi entrambi $i$ tubi di piombo alla medesima estremità del tubo di vetro, fu notato in entrambi un riscaldamento uguale. Ne deduce l' $\Lambda$. che un tubo più sotlile ed uno più grosso sviluppano nell'unilà di sezione la slessa quantilà di calore se l'ampiezza di oscillazione è uguale per tutti e due; e che il tubo più soltile nell' esperienza precedente si riscaldava di più soltanto perchè quest' ampiezza era maggiore, come appunto 
132

indicava la maggiore intensità del suono reso dal sistema.

Diedero risultati simili dei tubi di piombo che rendevano un suono proprio. Dopo lungo e forte strofinare

un tubo di $16^{\mathrm{mm}}$ diam. esterno, al nodo non dava deviazione

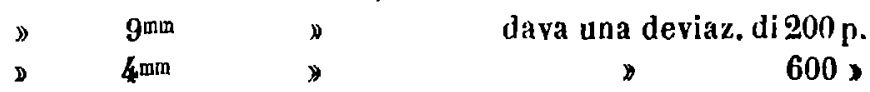

I.a lunghezza, ela grossezza della parete erano uguali pei 3 tubi. Diminuiva la deviazione collo scostarsi dal nodo, ed ai ventri non si potè scorgere quasi alcun riscaldamento. Non v' ha dubbio che il riscaldamento maggiore dei tubi più sottili si spiega dalla ampiezza di oscillazione, la quale in essi deve esscre maggiore che nei piu grossi, mantenendo costante la forza che causa il suono.

Giunse l'A. a riscontrare un aumento di temperatura anche in altri metalli, impiegandoli però in forma di fili sottili congiunti ad un tubo di vetro. Un filo d'ottone della grossczza di quasi $2 \mathrm{~mm}$ e della lunghezza di mezza onda del suono reso dal tubo, diede al nodo una deviazione di $100 \mathrm{p.;}$ accorciando il filo, si ottennero 300 p. Si succedono per l' entità del riscaldamento osservato: il rame, il ferro, l'acciaio, il legno.

E nota la proprieta del caoutchouc di ammorzare potentemente i suoni; a l' A. osservò un riscaldamento corrispondente a più di $1000 \mathrm{p}$. di scala in un piccolo tubo di caoutchouc adattato al solito tubo di vetro. Un termometro postovi a contatto prima del suono scgnava $19^{\circ}$ e dopo ne segnava 21.

Mentre pei tubi di altra sostanza, nei quali si formano più nodi, il riscaldamento è pressochè uguale nei diversi nodi; esso non si manifesta pel caoutchouc che ad una piccola distanza dal punto di congiunzione col tubo di vetro. Questo interessante risultato dipende, secondo l' $\Lambda$., dall'indebolirsi che fa il suono attraverso il caoutchouc cosicche ben presto non ha intensità suffciente per produrre un riscaldamento sensibile.

ll solo corpo, in cui l'A. non potè riscontrare un aumento di temperatura, i il vetro. I tubi sottili, posti a vi- 
brare fortemente, si spezzavano, e nei grossi non si poteva forse notare alcun riscaldamento, perchè non si facevano vibrarc con forza sufficiente.

Suoni trasversali. - Le successive condensazioni e rarefaziodi che si producono durante isuoni longitudinali sono una condizione necessaria affinchè il corpo sonoro si riscaldi. Ora siccome anche nci suoni dovuti ad oscillazioni trasversali avvengono delle coudensazioni e delle rarefazioni, cosi era da aspettarsi un riscaldamento anche in questo caso. Infatti l'A, lo coustato, tha trovd una distribuzione del calore prodotto molto più complicata che nel caso dei suoni longitudinali. Si servì di un corista ad un braccio del quale aveva sul prolungamento di esso adattato o un filo, o un cannelfo costituito della sostanza da esaminare. Con questo processo riscontrò un aumento.di temperatura nel caoutchouc, nel piombo, nell' ottone, nel rame, nel ferro, nell'acciaio; e per le diverse materie trovo valori corrispondenti a quelli ottenuti dai suoni longitudinali. Tuttavia nel caso delle oscillazioni trasversali riscontro in generale la stessa temperatura nei ventri che nei nodi; ed anzi pel caoutchouc la temperatura era maggiore nei ventri; all' estremità libera il riscaldamento era nullo per tutti $i$ diversi corpi. E da quest' ultima circostanza 1 'A. è condotto alla spiegazione del fenomeno; dice cioè che i luoghi di maggior flessione i quali nelle verghe liberamente vibranti si approssimano ai ventri, sono anche luoghi di riscaldamento maggiore; appunto come nelle oscillazioni longitudinali i luoghi ove maggiormente varia la densità, e che coincidono coi nodi, mostrarono maggiore sviluppo di calore. In modo corrispondente le esperienze di Kundt (1) indicano che l'azione delle verghe vibranti sopra la luce polarizzata che lo attraversa diminuisco avvicinandosi ai ventri nel caso delle vibrazioni longitudinali; e nelle vibrazioni trasversali diminuisce invece andando verso i nodi c verso l'estremilà libera.

L'A. riassume it risultati di queste ricerche dicendo:

1.0 Che ogni corpo solido, suonando si riscalda sensi-

(1) Poggend. Annalen, Vol. 123. 
bilmente, quando vi si succedano sufficienti condensazioni e rarefazioni; e che il riscaldamento cresce rapidamente insieme coll' intensità di tali condensazioni e rarefazioni.

2. Che il maggiore riscaldamento nelle sostanze che più ammorzano il suono, non dipende da una minore capacità calorifica, ma dal prodursi in esse maggior quantità di calore mentre vibrano.

3. Che si produce maggior quantità di calore in quei corpi nei quali il suono si propaga con minor velocità.

Ma con ciò l'A. non intende escludere che influiscano sul riscaldamento dei diversi corpi anche le differenze specifiche della maleria.

\section{A. RoIti.}

\section{Proposta d' un ulteriore porfezionamento} del microscopio; per J. B. LISTING (Nachrichlen $v$. $d$. K. Gesellschaft d. Wissenschaften zu Göllingen, 1869, n. 1)-

Gli eccellenti microscopi di E. Hartnack, che ottennero il premio massimo all' esposizione di Parigi, indussero l' $\Lambda$. a sottoporre a nuovo esame un principio diottrico della costruzione del microscopio, che già aveva in vista da molto tempo.

Nei microscopii fin qui usati non si forma che una sola imagine reale, dalla quale poi risulta quell’imagine virtuale che si presenta all'occhio. Ora la proposta dell' $A$. introdurrebbe due imagini reali e cosi l' istrumento effettuerebbe tre successivi ingrandimenti parziali invece di due.

L'A. spiega la nuova disposizione del microscopio col dire che al comune oculare composto di due lenti si deve imaginare sostituita una combinazione, quale è nella maggior parte degli oculari a quattro leati dei cannocchiali terrestri. Allora una delle due imagini reali accennate si trova davanti alla prima lente e l'altra fra le due ultime. A qresto oculare accoppia l'A. uno dei nuovi sistemi obbiettivi di Hartnack.

In tal guisa egli ottenne degli aumenti nell' ingrandimento di $20,28,55,97$ e 137 per cenlo confronto all' ingrandimento (che è 300 ) dato dall' obbiettivo n. 7 combinato coll'oculare n. 3 di Ilarlnack ad una distanza da esso di 20 cen- 
timetri. Il potere penetrante sarebbe aumentato anch' esso circa nella medesima proporzione, ed anzi l' $\Lambda$. nota che questo confronto delle osservazioui fatte col suo metodo e quelle fatte nel modo fin qui usato mette in evidenza l'alto grado di penetrazione di cui sono forniti i nuovi obbieltivi di Hartnack.

Dà poi delle istrazioni a quei costruttori che volessero seguire la sua proposta affinchè preferiscano alle lenti semplici, pei duc primi membri del sistema oculare, delle combinazioni acromatiche aventi l'apertura di 150 a 20 millimetri ed un diafragma interposto della largheza di 6 a 9 millimetri ed affinchè ai dıe ultimi membri diano essenzialmente la solita disposizione dell'oculare di IIuyghens. Quanto alla lunghezza del tubo dice che per l'ingrandimento del 97 per cento ne usava uno lungo 420 millimetri ed osserva che anche se fosse di 500 millimetri non sarebbe ancora incomodo specialmente dandogli la posizione obbliqua com'è d'uso nei microscopi inglesi.

Per dare un esempio d'ingrandimento suppone un sistema obbiellivo avente la distanza focale di 1 millimetro. Se la prima imagine reale si presenta alla distanza di $200 \mathrm{mil}-$ limetri dal secondo punto principale di questo sistema, e se il sistema oculare considerato da per sè rappresenta un microscopio che ingrandisce 280 volte, l'ingrandimento della combinazione sarebbe 5000, essendo la lunghezza del tubo 400 millimetri.

1 punti cardinali diottrici di un microscopio sono ordinati in uno degl' istrumenti fin qui usali come in una lente concava. Indicando con $\mathbf{E}, \mathbf{E}^{\prime}$ i due punti principali di tutto il sistema, con $F, F^{\prime}$ i due fuochi, con $G, G^{\prime}$ i due punti secondari; questi punti stanno sull' asse ottico nell' ordine EFH $\mathbf{G}^{\prime} \mathbf{F}^{\prime} \mathbf{E}$ cd è $\mathbf{E} \mathbf{F}^{\prime}=\mathbf{F G}=\mathbf{G}^{\prime} \mathbf{F}^{\prime}=\mathbf{F}^{\prime} \mathbf{E}^{\prime}=$ alla distanza focale negativa dell'intero sistema, ed il cosiddetto interstizio $\mathbf{E} \mathbf{E}^{\prime} \mathrm{e}$ circa uguale alla lunghezza totale dello strumento. Nel caso dell ingrandimento 400 (prendendo per base la distanza della vision distinta a 200 millimetri dall'occhio) la distanza focale di tulto il microscopio sarebbe negativamente di $0^{\mathrm{mm}}, \mathrm{o}$. Invece per un istrumento avente la nuova disposizione l'ordine 
136

di quei punti sarebbe GFE $E^{\prime} F^{\prime} G^{\prime}$, e la distanza fucale $F E$ sarebbe positiva. Per l'ingrandimento 5000 la distanza focale di tutto il microscopio risulterebbe $\mathrm{di}+0^{\mathrm{mm}}, 04$. $\Lambda \mathrm{l}$ sistema obbiettivo dovrebbe darsi nel caso precedente la distanza focale di 3 millimetri ed in questo caso di 1 millimetro.

\section{A. RoITI.}

Intorno ad un metodo possibile per vedere lo protuberanze rosee quando non vi ò ec. eliase; del sig. GUGLIELYO IIUGGINS. (Dal Monthly Nolices of the R. A.S.) - Nel rapporto del mio Osservatorio al termine dell'anno decorso fu messo in chiaro che enegli ultimi due anni - erano state fatte numerose osservazioni allo scopo di poter

- vedere le protuberanze rosee che si mostrano durante un - ecclisse di sole. Se questi corpi fossero gassosi il loro spet- tro consisterebbe di linee lucide. Con un potente spettro- scopio la luce riflessa dalla nostra atmosfera vicino al lem- bo del sole, sarebbe grandernente ridotta in intensità per - la dispersione dei prismi, mentre le linee lucide delle pro- minenze, se ve ne sono in vista, rimarrebbero soltanto di - poco diminuite in splendore. Questo principio è stato messo - in evidenza da apparecchi prismatici di varie forme ed - anche variamente disposti, ma fin ora senza successo .. Je osservazioni dell' ecclisse dell' Agosto passato avendo mostrato la posizione nello spettro delle linee lucide delle protuberanze rosee, il sig. Lockyer ed il sig. Janssen riuscirono indipendentemente l' uno dall' altro con un metodo analogo a vedere gli spettri delle protuberanze medesime.

a Il mio scopo è di descrivere in questa nota uno degli * apparecchi ricordati in quol rapporto ».

Esso si componeva di diaframmi di vetri colorati e di altri mezzi assorbenti, coi quali io poteva isolare una porzione dello spettro. Sembrava molto probabile che se le parti dello spettro, le quali sole allora rimanevano, fossero state identiche con quelle nelle quali $s^{\prime}$ incontrano le linee lucide delle protuberanze, queste sarebbero state visibili.

Per questa ricerca io adoperai una gran varietà di vetri colorati, e di altri mezzi assorbenti. In primo luogo li esa- 
minai con un prisma per conoscere il potere assorbente che essi avevano sulle diverse parti dello spettro: poi li cambiai :u vari nodi. Questi vetri furono soventi volte impiegati davanti all' occiaio, ma più frequentemente proiettando l'in. magine del lembo del sole su un diaframma, dopochè la luce era passata atíraverso dei mezzi colorati. Nel fare questi esperimenti si operò in manicra da liberarsi dall' intera immagine del sole; affinchè l'occhio rimasto in una oscurità relativa, potesse esser più sensibile alla luce assai debole delli ogge!ti cercati.

Siccome io non aveva cognizione della posizione nello spettro delle linec luvide, sarebbe stato soltanto per caso se io fossi riuscito a pote: vedere le protuberanzc

Ora che le posizioni di queste linee sono conosciute, questo metodo sembra p:ometter molto. Forse la luce intorno alla linea $\mathrm{C}$ de' russo si potra più facilmente isolare. Io ho un veiro losso capo il quaic assorbe tutto lo spettro eccetio it rosso esiremo. Dopo ie osservazioni dell' India io ho potuto soltanto fare un tenlutivo, mentre lo stato dell' atmosfera cr'a sfavorevole.

Egli è chiaro però che con questo metodo la forma c l' apparenza dolle protrheranze potrebbero essere osservate, e misurate accuratamente.

Nota del sig. NORMANNO LOCKYER rolativa ad uno scritto del sig. HuGGISS \& INTORNO AD UN POSSIBILE METODO PER VEDLRE LE PROTUBERANZE ROJEE QUANDO NON Vl ì ECCL!sse. - (dal Monthly Notices of the R. A.S.) - Nello scritto sovraccennato io leggo ciò che segue:

- Le osservazioni deli' ecclisse de!l'Agosto passato avendo - mostrata la posizione nello spettro delle linee lucide delle - protuberanze rosee, il sim. Iockyer ed il sig. Janssen rilla scirono indipendentemente l' ano dall' altro con un metodo analogo a rendere gii spettri dolle protuberanze medesimes.

La più semplice iuterpeirizione di questo paragrafo è che la mia scoperta si basò sulle osservazioni fatte durante l'ecelisse nell' India, e che il mio successo fu dovuto alle medesime.

Serie. 2. Yol. II. 


\section{8}

Io perciò chieggo il permesso di provare: $10^{\circ}$ Che il mio lavoro non si basó sulle osservazioni dell'India : $2 .^{\circ}$ Che il mio successo non fu dovuto per verun conto alle medesime.

I. E primieramente io posso osservare che il mio lavoro fu incominciato nel 1866 , e che il metodo da me seguito $\mathrm{fu}$ con chiarezza esposto in uno scritto comunicato nell' anno stesso alla Società Reale.

Le unic ossorvazioni furono allora senza successo a ragione del piccolo polere dispersivo dell' istrumento impicgato, il quale come io mi sono dipoi accertato, era affatto incapace di far vedere le linee lucide.

Secondariamente - Io mi provai di rimediare a questo stato di cose col far uso di uno strumento di maggior potere dispersivo, e ottenni un sussidio per la costruzione di un tale strumento, che fu ordinato nel Gennaio 1867.

In terzo lnogo - Dopo una lunga serie di malaugurati ritardi dovuti ai costruttori dell' istrumento, e ad una mia grave malattia, $l$ istrumento fu ricevulo in uno stato incompleto il 16 0itobre decorso (Venerdi notte).

In quarto luogo - La scoperta dello spettro delle protuberanze fu falta il 20 ottobre (Marledi mallina) cioè quattro giorni dopo aver ricevulo $\mathrm{l}^{\prime}$ istrumento, e precisamente nel primo giorno che io consacrava alle osservazioni, dopochè lo strumento fu montato, rettificato e provato.

In quinto luogo - Il mio actodo di osservazione cra di dirigere il mio telescopio su una porzione speciale del lembo del sole, e quindi di scorrere lungo lo spettro molto lentamente dal rosso al violetto, poi di dirigerlo su un'altra parte tenendo lo stesso sistema e quindi incominciare di nuovo.

II. Veniamo adesso al secondo punto - In ciò che ho fin quì esposto ho dimostrato che la coincidenza in tempo fra $i$ miei resultati e il ricevimento delle informazioni dall' India era dovalo alla data nella quale il nuovo istrumento era stato ricevato. Io sono convinto che se il nuovo spettroscopio fos. se stato ricevato un anno avanti, la scoperta sarebbe stata fatta colla stessa faciliti: e le considerazioni del P. Secchi sulla possibilità delle osservazioni, anche con uno strumento di moderato potere dispersivo, convalidano questa opinione, Io aveva di più stabilito il mio metodo d'osservazione. 
Adesso vediamo quanto contribuiranno al mio successo le osservazioni dell India, o se in realta esse potessero aver cagionato il successo di chi ne avesse avuto bisoguo.

Nel 20 Ottobre io conobbi tre serie di osservazioni, quello di Raget, di Hersehel o di Tennant.

Raget dette B D E C F. e di più quattro linee indeterminate.

$$
\text { Herschel dette }\left(\begin{array}{c}
\text { vicino } \\
\text { a } \mathrm{B}, \mathrm{o}
\end{array}\right) \text { D }\left(\begin{array}{c}
\text { vicino } \\
\text { ad } \mathrm{F}
\end{array}\right)
$$

osservando non essere affatto la F, ed esitando moltissimo ad assegnare un posto anche approssimato alla linea nel rosso.

$$
\text { Tennant dette C D b }\left(\begin{array}{c}
\text { vicino } \\
\text { a F }
\end{array}\right)
$$

egli credette altresi aver veduta una linea presso G.

La tavola precedente mostrera, io penso, come le osservazioni Indiane furono affitto inutili per ciò che asserisce il sig. Huggins. Infatti vi sono mentovate tutte le linee dello spettro distinte con lettcre da Fraunhofer tranne la A ed II. So io mi fossi servito di esse certo avrei naluralmente cereala la linea $\mathrm{D}$, siccome la sola nella quale lutte le osservazioni si trovavano d'accordo. Io mi riferii chiaramente all' idrogeno (che dà le linee $(\mathrm{C}$ ed $\mathrm{F}$ ) nel inio scrillo del 1866; ma nel 1868 soltanto un osservatore è sicuro di C, e soltauto un osservatore ò sicuro della $F$, e ciò nonostante si dice che il mio successo d dovuto alle osservazioni indiane.

Io ho mandato questo foglio alla R. Socicta astronomica non perchè la lode dovuta al sig. Janssen e a me stesso sia impiccolita dallo scritto del sig. Huggins, tna perchè io so, per esperienza, che le Nolizie Mensili sono una delle pii importanti sorgenti dalle quali deriva la storia dell'Astronomia.

Io naturalmente preferisco che una relazione veridica della recente scoperta, apparisca nelle Nolizic Mensili, invece di un esposizione inesatta del fatlo, la quale se fosse lasciata senza osservazione, ingannerebbe quelli che verranno dopo di noi. 


\section{0}

Sulla polarizzazione della luce axznryognola doll acqua; per L. SORET (Bioliolheque de Ge. neve, Mai 1869). - A cagione d'altre rice.che sono stato condotto ad occuparmi d' una quistione, che si è molte volte discussa, quale è quella, di sapere se la colorazione azzurra dell'acqua è dovuta al liquido stesso o a particelle solide che vi stiano sospese. Un lavoro recente di Tyndall sulla polarizzazione della luce del ciclo, e le sue belle esperienze sulle proprielà oftiche delle sostanze allo stato di nube (1) $\mathrm{mi}$ hanno suggerito l'idea che se la tinta azzurra dell' acqua è prodolla almeno parzialmente da particelle solide in sospensione, questa colorazione deve esser accompaguata da fenomeui di polarizzazione simili a quelli che carallerizzano la luce del cielo. Questo è difatti ciò che io ho osservato sull'acqua del lago di Ginevra, il bel colore della quale e ben noto, e senza pretendere di giungere ad alcuma conclusione, mi sembra che questa analogia fra la luce del cielo e quella dell'acqua presenti qualche interesse (2).

(1) Archives, Febbraio 1869, T. 36, pag. 156.

(2) Fu mia inteazione di stabilire anzitutto in questo brere ragguaglio l'analogia che esiste fra la luce del cielo e quella dell'acqua al puato di vista della polarizzazione; poichè non so che alcuno prima di me abbia osservato questo fatto. Le conseguenze poi che si potrebbero trarre sulla colorazione sarebbero forse premature. Tuttavia i fenomeni di polarizzazione che descrivo qui mi sembrano favorevoli alla ipotesi gia vecchia che quella colorazione sia dovata almeno in parte alla presenza di particelle solide piccolissime sospese nell' acqua.

Il giornale l'Institut (nel n. ${ }^{0}$ del 21 Aprile 1869) fa la seguente obbiezione su quell argomento. - Questo modo di vedere non sembra che - s' accordi col fatto che la colorazione azzurra è lanto più intenisa, quan- to più l'acqua è calma e limpida a. Vi si puó rispondere citaudo lo belle osserrazioni di Tyndall, che sono state il punto di partenza delle mie ricerche : Esse ci apprendono che nei gas, è soltanto quando le particelle sospese sono estremamente tenui, che vi è colorazione azzurra e polarizzazione completa; se le particelle sono piú grosse, la colorazione diventa bianca e i fenomeni di polarizzazione scompaiono o si modificano assai. Non si potrebbe forse presumere per analogia che più l'acqua è limpida, cioè quantopiù le particelle sospese sono piccole, più la colorazione azzurra dev'essere sensibile? Ora poi $\dot{e}$ a questa sola causa, che si deve atattribuire la tiuta azzurra doll' acqua $P$ Questo è ció che io non potrej assicurare con sicurezza.

Secondo lo stesso namero dell' Instilut Dumas, che ba avulo la gen- 
L'apparecchio del quale mi sono servito per verificare questo taito si compone semplicemente d' una specie di cannocchiale, di cui l'obbicitivo è formato da una lama di vetro a faccie paralele, che chiode ermeticamente il tubo del cannocchiale in guisa che si possa immergerne l'estremit a nell' acqua, senza che essa ent: $i$ nell' interno. $L_{\text {' }}$ oculare è formato da un prisma di Nicol. Si comprende che introducendo nell' acqua l' esiremià oggeíiva del cannocthiale, l'occhio situato all' altra estrenità riceve la luce a zurra emessa dall' acqua, e che facendo girare il prisma di Nicol si può analizzare quella luce e riconoscere se è polarizzata.

I raggi su'ni cadenoo sulla saperficic dell' acqua vi penetrano restando paral'eli gli rai acki aliri, se almeno la superficie di separazione cei due mezzi è perfettamente piana, cioè se il tempo è calmo. Dunque se, mettendosi in un battello, si dirige l' asse del cannocchiale, perpendicolarmente alla direzione di quei raggi refratii, ci troviamo nelle condizioni analoghe a quelle nelle quali si osservi il massimo di polarizzazione della luce del cielo, cioè quando si guardano ad angolo retto col sole.

Operando in questo modo in un luogo ove la profondità del lago era sufficiente per non vedersi il fondo, io ho riscontrato una polarizzazione ben cistinta. Il piano di polarizzazione passa per l'asse del cannocchiale e il sole (1).

Quanto più s'allontana il cannocchiale dalla direzione

tilezza di presentare la mia nota all'Accademia, vi ha aggiunto l'osservazione interessante che nei bacini di Menilmontant dove effluiscono le - acque del Dhuys si pnò osservare la colorazione azzarra che presenta - l'acqua dei laghi della svizzera. I'acqua di quei bacini è chiarissima, - traspareníssima, e presenta una tinta azznrra molto intensa *. 10 crodo difatt, che la colorazione azzurra debba appartenere a cutte le acque sufficientemente limpide, so non contengono disciolte delle sostanzo colorate. So non m' in 'aano il Saint clair Devillo ha rimarcato cho le acque, delle quali il residuo dell'ercporazione è bianco, ha nno una tinta azzurra, - che quelle, il residuo dello quali é giallo, hanoo una tinta verde.

(1) Dopo le prime osservazioni riporta:? poc' auzi io ho irovato, che interponendo una lama di quario sul pussaggio dei raggi, prima che essi ạrrivino al prisna di Nicol, si puó riscontrare la polarizzazione della luce, mediante la colorazione ben netia che si manifesta. 
142

perpendicolare ai raggi solari, altrettanto meno è spiccata la polarizzazione, e non tarda wolto a scomparire completamente. Nullameno però io non ho potuto sin qui determinal'e con esattezza se veramente si ottenga la massima polarizzazione quando l'asse del cannocchiale ed i raggi solari fanno fra loro un angolo di $90^{\circ}$.

Si comprende quindi che in questo caso il fenomeno è più complicato che per la luce del cielo.

Primieramente d evidente che se la superficie dell' acqua è agitata i raggi solari dopo la loro refrazione non sono paralleli; per conscguenza il fenome oo sarà tanto meno morcalo, quanto più l'acqua è agitata. Questo appunto, è quello che ho notato.

La prima volta che ho provato il mio apparecchio siccome il vento era alquanto impetuoso, io non ho potuto vedere traccia alcuna di polarizzazione, la qual cosa poteva forse dipcndere dall'aver spirato nei giorni precedenti un violentissimo vento di tramontana, e che per questo l'ácqua fosse un poco torbida. In altri duo giorni d'osservazione il lago essendo pochissimo agitato, la polarizzazione era ben distinta. Finalmente un giorno di calma quasi perfetta essa era tanto manifesta quanto quella del cielo, che a dir vero, in quell' ora non era troppo azzurro.

In secondo luogo i raggi che sono penetrati nell acqua sono parzialmente polarizzati per refrazione, ed è facile vedere che quando l'asse del cannocchiale è nel piano verticale che passa pel sole, cioè nella posizione più comoda per l'osservazione, i raggi già polarizzati per refrazione devono cssere estinti anzichè riflessi dall' acqua (l).

Finalmente la luce solare diretta, non è la sola che arrivi all'acqua: vi si aggiunge la luce diffusa che arriva da tulte le direzioni, e che quando è riflessa dall' acqua deve produrre un infinita di raggi polarizzali in piani differenti. Allorchè il cielo era coperto di nubi io non ho potuto riscontrare alcun indizio di polarizzazione.

(i) Come nella sperienza di Tyndall, nella quale una nube azzurra rischiarata da un fascio di luce giả polarizzata. (Arohives, loogo citalo, pag. 105. 
Intanto nutro viva fiducia di poler completare queste mie osservazioni o coll' uso di una luce artificiale, o coll' estenderle ad altre acque che quelle del lago di Leman (1).

r. G.

\section{Dell'intensita della corrente nella macchi-} na elettrioa; per I. POGGENDonfF. ( Annali di Poggendorff, T. CXXXIV ). - In:una serie di esperienze precedenti a quelle, che riguardano questo nostro soggetto, l'A. dimostrò, che quando la corrente è costretta a bilorcarsl fra due tubi simili, dell Iloltz, paralleli e disposti in senso contrario, essa non attraversa che il tubo positivo; e quando si adoperi la macchina stessa dell' IIoltz, questo risullamento è costante e non può esser modificato neppure dall' addizione delle più forti resistenze nel circuito del tubo positivo. Questo fatto secondo l'A. porterebbe a credere che l' intensità delle correnti di tal genere non dipenda dall'influenza. Dall' opera del Gauss stampata nel 1837 col titolo di Osservazioni magnetiche, rilcvasi come egli pure fosse giunto ad una risultanza identica, poichè imitando l' esperienza del Colladon, ciod congiungendo un galvanometro ben isolato, ed a sistema pressochè completamonte astatico, egli trovó, che l'intensità della corrente non variava per l'aggiunta della resistenza nel circuito, e concluse altresì, che l'azione elettromagnetica della corrente della macchina clettrica, era indipendenle dalla resistenza, e che per questa proprietá caratteristica, essa distinguevasi da tutte le altre.

Noi sappiamo difatti, che l'intensitit di una corrente non c̀ altro cho la quantità di elettricità che passa nel conduttore nell'unità di tempo, e che nelle correnti dette galvaniche, cioè dovute all'azion chirnica, essa varia con questa, e questa alla

(1) In aloune esperienze rocentissirre io bo riconosciuto, che un fascio di lace diretto attraverso di una massa d'acqua (acqua del lago portata dai condotti della cilta di Ginerra, anche quando e filtrata) vi pro. duce na traccia luminosa che presenta una polarizzazione rimarchevole, - moltissimo somigliante a quella che si mauifesta nei gas cho tengono sospesi doi corpicciuoli. Ty ndall area già osservato prima di mo questo fenomeno sopra diversi liquidi; o mi area reso arvisato con una sua lettera particolare. 


\section{4}

sua volta varia colla resistenza; ma ciò non avviene quando usasi come sorgente una macchina oleirica, giacche allora la quanlita di coltricita non dinende cho di certe condizioni della macchina siessa, o tutto finise nella sciatilla, qualunque sia del resto la lm: ghezza del conüliore.

Quest'esperienza del Causs concor'ava con quelle di altri esperimentaiori abilissini; ma nulladimeno si esigeva una riconferra, e questa ce la diede poco fa il soszendorff, quando facendo uso di una macrinina dell' Holtz o di un buon galvanometro, egli trovò che la deviazione era costante, tanto se il conduitore che congiungeva quesii due apjareccli era assai resisiente, quanto se nou lo era che pochicsimo. Típeiè pure la stessa esperienza con una altra macolina a 90 aprrture, ed i risuliamenti non smeníirono quel prino fatto; nè pure egli ebbe risulianze contrarie usando la bottigia di Leida, nè coi processi tors umeirisi. Si può dunque concl-dere coll' A. clie tanto lo sviluppo dell' elcttricita, quanto 'lintensiti delle correnti prodolte cualle macnhire eleitriche è indipendente dalla resistenza come lo avevano già detto Ampère e Gauss.

I. G. 